\title{
Los suplementos especializados como guetos de noticias en la prensa generalista: el caso de los científicos y sanitarios
}

\author{
Dr. Carlos Elías \\ Profesor titular interino de la Universidad Carlos III de Madrid
}

\section{RESUMEN}

En este estudio se ha analizado el impacto que tiene en la publicación de noticias de ciencia y sanidad la existencia de suplementos especializados de esas áreas en los diarios generalistas españoles de tirada nacional. Para ello se ha realizado un estudio cuantitativo entre diarios que sí tienen esos suplementos con otros que no los publican y que incorporan esas informaciones en la sección de sociedad. La conclusión es clara: la existencia del suplemento provoca que se incluyan más noticias de esa área en el diario pero también que se postergue su publicación y no aparezcan el día en el que son noticia sino que se aguanten hasta la fecha de publicación del suplemento. Esto, obviamente, satura al lector medio y provoca que la información tenga menos impacto convirtiendo los suplementos en guetos de noticias especializadas y evitando que éstas aparezcan en sociedad o en primera página (puesto que normalmente son noticias ya conocidas). En este sentido, el estudio intenta abrir el debate sobre la conveniencia o no de determinados suplementos especializados.

\section{ABSTRACT}

In this study there has been analyzed the impact that has in the publication of news of science and health the existence of specializing supplements of these areas in the spanish newspaper sold in all the country. For it, a quantitative study has been realized among diaries that have these supplements with others ones that do not publish them and that incorporate these type of informations in the section of society.

The conclusion is relevant: the existence of the supplement prompt that are included more news of this area in the diary but also that postpone its publication and they do not appear the day in which they are a news but they restrain themselves up to the date of publication of the supplement.

This, obviously, saturates the average reader and provokes that the information has less impact, turning to the supplements in guetos of specializing news and preventing these news appearing in society or in the first page (since normally they are news already known). In this respect, the study tries to open the debate on the benefit of specializing supplements.

Palabras claves: Periodismo científico/Periodismo sanitario/Suplementos especializados/Sociedad.

Key words: Scientific journalism/Sanitary journalism/Specializing supplements/Society. 

generalista: el caso de los científicos sanitarios

\section{Introducción}

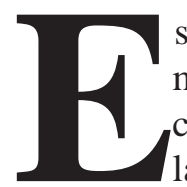

s importante advertir que en un sistema tan dinámico como el periodismo, los datos varían con mucha frecuencia por lo que un estudio cuantitativo no tiene más valor que el de indicarnos por dónde se mueve la realidad. Tal y como ya sugería Blumer ${ }^{1}$ en 1954, no puede reducirse la realidad a variables numéricas. Por ello, más que los datos, lo importante son las tendencias, si éstas se consolidan y qué significa eso en el contexto del periodismo.

Para llevar a cabo un estudio sobre el impacto de la existencia de los suplementos de ciencia y sanidad en la publicación de estas noticias en la prensa generalista era necesario, en un primer paso, contabilizar todas las noticias sobre estas áreas publicadas en un periodo determinado y, después, clasificarlas en función de si habían aparecido en suplementos o en la sección de sociedad. El período de tiempo fue de febrero a julio, ambos inclusive, de 1998 y los periódicos: El País, El Mundo, Abc, La Vanguardia, El Periódico de Cataluña y Diario 16.

Una vez establecidos los criterios para seleccionar las noticias ${ }^{2}$ se hallaron en total 1.458 noticias sobre temas científicos, incluyendo en éstos también los medioambientales y los sanitarios. Observamos que 758 de ellas proceden de suplementos de ciencia ${ }^{3}$ o de salud. Esta cantidad supone que el 52 por ciento de las noticias científicas que se publican en los seis periódicos españoles tomados como referencia aparecen en sus suplementos. Sin embargo, debe matizarse que no todos los periódicos estudiados disponen de suplementos específicos. Así, durante el período estudiado del año $1998^{4}$, de los seis diarios estudiados -El País, Abc, El Mundo, La Vanguardia, El Periódico de Cataluña y Diario 16-, sólo tres -El País, Abc y El Mundo- disponían de suplementos. El País publicaba uno sobre salud los lunes y otro de ciencia los miércoles; El Mundo publicaba uno de salud los jueves y dos páginas de ciencia en el suplemento Crónica de los domingos, y el diario $A b c$ incluía su suplemento de ciencia en el $A b c$ Cultural publicado los viernes.

Contabilizamos que en esos tres diarios aparecía, en el periodo estudiado, un total de 1.102 -el 75 por ciento del total-; teniendo en cuenta que fueron 758 las que procedían de sus suplementos, se concluye que el 68,8 por ciento de las noticias científicas publicadas por esos tres diarios aparecían en sus suplementos.

1 BLUMER, H.: "Whats is wrong with social theory". American Sociological Revew, n 19. 1954.

2 ELÍAS, Carlos: "Estudio cuantitativo de las fuentes en el periodismo español especializado en ciencia" en Revista Latina de Comunicación Social, Universidad de La Laguna, no 38 (febrero de 2001).

3 Hemos considerado como suplemento de ciencia las dos páginas semanales publicadas en el suplemento dominical Crónica de El Mundo.

4 En la actualidad se han modificado los criterios editoriales y han aparecido algunos suplementos nuevos, mientras que otros se han dejado de publicar. 
Este porcentaje superaba el 75 por ciento para el caso de El País y El Mundo. En concreto 78,6 por ciento para el primer diario y 77 para el segundo. Lo que demostraba que en estos dos diarios los suplementos se han convertido en el gueto o baúl donde van a parar las noticias científicas o relacionadas con la salud.

El caso de $A b c$ resultó curioso, pues debido a que publicaba diariamente una sección dedicada a la ciencia, el porcentaje de noticias de suplementos se redujo a un 50 por ciento. Está claro que ese resultado posiblemente no se repita en otros períodos y que sea simplemente coyuntural. Sin embargo, el obtenido en los meses estudiados refleja que este diario es el único que realiza un reparto adecuado en las informaciones.

En la clasificación por noticias, el primer diario es El Mundo con 404 informaciones sobre ciencia o salud. Sin embargo, la inmensa mayoría de ellas, 311 (77 por ciento) procede de su suplemento de salud -en nuestro período se incluyeron 26 suplementos- que contaba con 8 páginas -el mayor de todos los publicados en España, llegó a contar en 1999 con 12 páginas, aunque ahora vuelve a tener 8- o de las páginas del suplemento dominical, de los cuales incluimos 25. El Mundo sólo publicó 93 noticias de ciencia en su sección de sociedad. Esta cantidad supone una noticia cada dos días.

Le sigue $A b c$ con 357 noticias, de las que 179 proceden de su suplemento -se estudiaron 26- y 178 de la sección ciencia que publica casi diariamente este periódico. Esta cantidad representa una media de casi una noticia diaria $(0,98)$, aunque en realidad muchos días publican incluso dos y hasta tres -las noticias breves que también publica este diario no las hemos contabilizado- y otros, especialmente los domingos y lunes, no suele incluir ninguna.

En tercer lugar se situó El País, con 342 noticias, de las cuales 184 proceden de los 27 suplementos estudiados de Futuro y 84 en el de Salud. Sólo publicó 73 informaciones de ciencia en sus páginas de sociedad, lo que representa el 21,4 por ciento del total de noticias publicado por este diario, lo que significa que El País publica dos noticias de ciencia cada cinco días: la proporción más baja de todas.

En cuarto lugar se situó el diario La Vanguardia, con 153 informaciones científicas, lo que implica casi una diaria en el periodo estudiado, tras él Diario 16, con 110 y El Periódico de Cataluña, con 93.

Si utilizamos como criterio el número de informaciones científicas de actualidad que se publica el día en que el hecho es noticia, tendremos que el primero es $A b c$ con 178, seguido de La Vanguardia, (153) y de Diario 16 con 110. El Mundo ocupa el cuarto lugar con sólo una noticia de diferencia respecto a $E l$ Periódico de Cataluña y, sorprendentemente, El País ocupa el último lugar en esta clasificación, con 73 informaciones.

Todo esto nos hace reflexionar sobre si el hecho de que existan suplementos ayuda o no a la difusión de la divulgación científica; sobre si resulta positivo 
sacrificar información científica del día en que ha sucedido y relegarla al día de publicación de los suplementos aunque, eso sí, tratada con mayor rigor. Y si para rellenar estos suplementos, a veces se exceden en la publicación de noticias provenientes de los gabinetes de prensa de las revistas científicas o de las instituciones oficiales pero que carecen de interés real.

\section{La progresiva desaparición de los suplementos de ciencia}

Los suplementos de ciencia y salud han aparecido en la prensa española como parte de una estrategia más en su lucha contra la competencia de los medios audiovisuales. A partir de los años 80 , los periódicos españoles se vieron en la necesidad de frenar la fuerte competencia, sobre todo con la televisión, y reaccionaron ante esta amenaza mediante dos estrategias diferentes: una reorganización del espacio ocupado por los distintos medios escritos y un aumento espectacular de producto informativo en cada ejemplar.

La reordenación del espacio ha tenido como primera víctima a las publicaciones de información general de periodicidad semanal y mensual, cuya función han asumido progresivamente los diarios de información general en sus dominicales. Además, estos diarios están asediando a las publicaciones especializadas, que se ven constreñidas a públicos cada vez más específicos. Sólo la prensa económica y deportiva mantiene con seguridad su propio espacio, pese a que los grandes diarios ofrecen suplementos económicos y deportivos que suponen una fuerte competencia.

La segunda estrategia ha propiciado que la prensa diaria de información general haya aumentado de forma espectacular no sólo la cantidad de noticias, sino la variedad de temas que ofrece. En este contexto hemos de inscribir el significativo aumento de la información científica en prensa española respecto a lo que se publicaba sobre ciencia a principios y mediados del siglo XX y donde se enmarca también la aparición de los suplementos.

Todas las encuestas han corroborado el creciente interés de los lectores por las noticias científicas. En este sentido, un estudio del Centro de Investigaciones Sociológicas (CIS) elaborado en $1998^{5}$ puso de manifiesto que el déficit informativo de la población española entre su interés por los temas científicos y las noticias que reciben a través de los medios de comunicación alcanza el 37 por ciento. Es decir, que mientras que un 63 por ciento de los encuestados mostraban "mucho interés" por la información científica en los medios de comunicación, sólo un 26 por ciento opinaba que se daba bastante información sobre estos temas. El estudio indicaba que el déficit se observa en asuntos relacionados con los avances médicos, con los descubrimientos científicos y con la ecología y el medio ambien-

5 Centro de Investigaciones Sociológicas, 1996. Estadística sobre grado de interés de la población española por temas. Estudio 2213. Datos y opinión. CIS , nº5. 
te. Los datos de la encuesta del CIS también revelan que no se observa este déficit en información deportiva y que respecto a la información política se detecta, incluso, un superávit de noticias publicadas de un 5 por ciento.

Sin embargo, la realidad hasta el año 2002, contrariamente a lo que podía esperar, no sólo por las encuestas sino por la evolución de los periódicos en el sentido ya descrito, no ha seguido las previsiones y los suplementos sobre ciencia y tecnología han disminuido. Así, periódicos como La Vanguardia en 1997 y Abc y El Mundo ${ }^{6}$ en 1999 suprimieron sus suplementos científicos.

La Vanguardia disponía en los años 80 de un suplemento de salud y de otro dedicado íntegramente a la ciencia y la tecnología, ambos independientes. Posteriormente se unieron para desaparecer definitivamente en 1997. El suplemento de La Vanguardia -dirigido por Vladimir de Semir- obtuvo, incluso, el premio nacional de periodismo científico y significó un hito en su momento, pues fue el primero que introdujo la infografía en color en sus reportajes científicos. Su desaparición provocó una avalancha tal de cartas de protestas al director y una reacción de las universidades de instituciones científicas catalanas que se pensó, incluso, en volverlo a publicar pero, finalmente, se decidió no hacerlo ${ }^{7}$.

El caso de $A b c$ quizá sea uno de los más tristes. En primer lugar porque fue el primer diario que incorporó la ciencia no a los suplementos de salud o de tecnología sino al suplemento de cultura. Luis María Anson fundó el suplemento Abc Cultural en 1991 y en 1993 incorporó la sección científica al mismo, con una filosofía revolucionaria en el panorama cultural español que mantenía que la ciencia era parte de la cultura y que ambas no pertenecen a mundos diferentes ${ }^{8}$. La desaparición de la sección de ciencia del suplemento cultural en 1999, que coincidió con la incorporación de Francisco Giménez Alemán como nuevo director, provocó también la protesta de muchas universidades españolas e, incluso, del presidente de la Real Academia de las Ciencias, Ángel Martín Municio, quien ha reprochado públicamente esta decisión.

En el caso de El Mundo las causas de la desaparición de las páginas de ciencia del dominical Crónica se deben a razones más prosaicas. El redactor científico que tenía El Mundo, Paco Rego, pasó a incorporarse como subdirector de la revista de divulgación científica Newton ${ }^{9}$ que pertenece al mismo grupo

6 En el caso de El Mundo, el suplemento de ciencia se publicaba los domingos dentro del dominical Crónica.

7 Esta información ha sido facilitada por fuentes del periódico La Vanguardia, que prefieren no ser identificadas en este trabajo.

8 En una conversación con Blanca Berasátegui, actual directora de El Cultural que se distribuye con El Mundo y responsable de $A B C$ Cultural en la época en la que Luis María Anson era director de ese diario, señaló que, en un principio, costó mucho convencer al mundo cultural español de que la ciencia pertenecía también al ámbito de la cultura y añadió que hubo muchas protestas de algunos sectores. Sin embargo, manifestó, el mundo científico y en especial las universidades sí fueron muy receptivos a esta idea.

9 La revista científica Newton dejó de publicarse a finales de 2001. Lo que podría entenderse como otro indicador de que la divulgación científica no tiene tanto mercado como algunos postulan. 
editorial que El Mundo y que comenzó a publicarse en 1998. Sin embargo, la razón principal para eliminar el suplemento fue la compra por el grupo editorial de El Mundo de la revista El Cultural que hasta septiembre de 1999 se distribuía con La Razón. A partir de esa fecha se distribuía con El Mundo y los responsables del suplemento Crónica consideraron conveniente eliminar las páginas de ciencia del mismo, para que no coincidieran los temas con los que aparecen en la sección de ciencia de la revista El Cultural $^{10}$.

En la práctica, esta decisión ha sido perjudicial para la divulgación de la ciencia española, puesto que El Cultural, debido a la falta de periodistas científicos en España, no ha conseguido una sección de ciencia del nivel que tenía el $A b c$ Cultural ni de las páginas del suplemento Crónica.

No he mencionado, hasta ahora, la desaparición del suplemento científico de El Periódico de Cataluña, porque ésta no se ha producido en estos últimos años, sino que tuvo lugar en 1991. Este suplemento, denominado $I+D$ estaba dirigido por Luis Ángel Fernández Hermana. En la actualidad, este diario catalán incorpora habitualmente en su suplemento dominical una página de ciencia, que elabora un colaborador del periódico pero no su redactor científico, Antonio Madridejos.

\section{Los suplementos de ciencia no convencen a los anunciantes}

En 2001, sólo El País de entre los grandes diarios nacionales mantiene su suplemento específico sobre ciencia.

Periódicos como La Vanguardia o Abc han justificado sus decisiones de prescindir de los suplementos científicos argumentando que prefieren publicar los artículos científicos en el día en que ocurren, tal y como lo hacen, por ejemplo, los diarios franceses, en especial Le Monde, el cual publica diariamente una página sobre ciencia en la sección Aujourd'hui Sciences, o algunos estadounidenses.

Pero los responsables de los diarios españoles se olvidan en sus comparaciones de que estos diarios europeos o estadounidenses publican cada cierto tiempo monográficos en los cuales hacen revisión del estado de un asunto científico concreto. Otro hecho que no tienen en cuenta los directores de la prensa española y que los científicos les reprochan es que en España, a diferencia de otros países europeos y, sobre todo de Estados Unidos, los investigadores no cuentan con una revista, tipo Science, que pertenece a la Asociación Americana para el Avance de la Ciencia, en la cual puedan escribir sus artículos de opinión o de revisión. Lo único que tenían eran los suplementos de ciencia y, ahora, sólo les queda el de El País.

10 La sección de ciencia de esta revista está íntegramente compuesta por colaboraciones de los científicos. Algo muy interesante y enriquecedor para la cultura española pues supone darle voz a los investigadores. 
Aunque los directores de los medios españoles no lo aseguren explícitamente, al parecer el problema principal radica en que la comunicación científica no ha logrado, contrariamente a lo que se esperaba en un principio, mover una publicidad específica hacia esas páginas. De hecho, El País apenas tiene publicidad en sus páginas del suplemento. Incluso algunas empresas tecnológicas no quieren anunciarse en las páginas de los suplementos científicos, debido a que no está nada claro que la ciencia tenga buena imagen entre la sociedad ${ }^{11}$.

Un estudio ${ }^{12}$ elaborado por Media Resource Service, una organización estadounidense que pone en contacto a periodistas con expertos en diversos campos, indica que en 1989 cerca de un centenar de periódicos de Estados Unidos poseían secciones específicas de ciencia. En 1992, esta cantidad había disminuido un 50 por ciento y en 1996, sólo 35 diarios estadounidenses tenían sección de ciencia. El resto, o la había eliminado o la había reconvertido en una de Health and fitness (salud y en forma).

Las causas de este descenso podrían estar, según el estudio, en el encarecimiento del papel de prensa, el escaso interés de las empresas en publicar anuncios en estas secciones, la disminución del nivel cultural de contenidos de los medios propiciada por las cadenas de televisión y la mala imagen que en un sector de la población tiene la ciencia, según algunos estudios sociológicos.

\section{El "sospechoso" aumento de los suplementos de salud}

Los suplementos dedicados a temas médicos sí han tenido gran aceptación, sobre todo porque los laboratorios farmacéuticos encuentran en ellos un magnífico lugar para publicitar sus productos, no sólo mediante anuncios directos, sino -y lo que es más grave- a través de las informaciones que aparecen en forma de noticias científicas pero que esconden un trasfondo de manipulación de los laboratorios farmacéuticos.

La perversión en las revistas médicas especializadas ha llegado a tal extremo que no es extraño encontrar titulares como: "Una gran revista médica pide perdón por evaluar fármacos con expertos pagados por sus fabricantes" (El País, 25-2-2000: 44).

En esta noticia, los responsables de The New England Journal of Medicine, una de las revistas especializadas más citadas, por ejemplo, en el suplemento

11 El periodista científico Jon Franklin, ganador del premio Pulitzer en 1985 y en la actualidad profesor de divulgación científica en la Universidad de Oregón (Estados Unidos), señala en un artículo publicado en la revista Quark, Ciencia, Medicina, Comunicación y Cultura (n¹1, 1998, pp. 53-63) que a partir de los años 70 la ciencia ha comenzado a tener una mala imagen ante la sociedad, la cual ha pasado de admirarla a casi temerla. En su experiencia personal, señala, es mejor no incluir la palabra ciencia o científico en el titular de una noticia si el periodista pretende que el redactor jefe le acepte el artículo y le conceda gran espacio, a no ser, añade, que el artículo considere aspectos negativos de la misma.

12 Citado en el editorial “QQuién mató la sección de ciencia?” en Quark, Ciencia, Medicina, Comunicación y Cultura (n' 9, pp. 4-6). 

generalista: el caso de los científicos sanitarios

Salud de El País, El Mundo y Abc, se disculpaban porque 19 artículos científicos publicados en los tres últimos años estaban escritos por investigadores que pertenecían a determinados laboratorios farmacéuticos, pero que lo habían ocultado. Los científicos autores de los artículos elogiaban las propiedades de determinados fármacos respecto de otros, en función de la financiación que percibieran de los laboratorios. Entre las empresas acusadas de emplear el método de "compra" de un investigador médico para que comentara, con apariencia de objetividad científica, las bondades de un fármaco en esta revista especializada que difundirá sus contenidos en la prensa mundial se encontraban: Roche, Glaxo Wellcome, Pfizer, Procter and Gamble, Bristol-Myers Squibb, Merk \& Co y Wyeth-Ayerst.

Los artículos "comprados" elogiaban los importantes resultados de determinados fármacos contra enfermedades como la hepatitis vírica, la meningitis y la osteoporosis.

En la denuncia no se menciona cuántos de esos artículos fueron citados posteriormente en la prensa, pero sí que esta práctica perversa puede ser más común de lo que en principio se piensa.

Desde el punto de vista de las disfunciones en el flujo de la comunicación científica con los periodistas, el problema estaría en que los medios de comunicación no contrastan una noticia proveniente de una revista como The New England Journal of Medicine porque se produce el fenómeno de la "noticia acatamiento" en el sentido descrito por Furio Colombo ${ }^{13}$. Este fenómeno se produce cuando la noticia llega a las redacciones con un plus de seguridad tal que el periodista baja la guardia de la comprobación. En los casos mencionados este fenómeno se ve favorecido por la "apariencia de credibilidad" que le da a una revista como la mencionada el estar asesorada por varios premios Nobel -una circunstancia que cita de forma expresa en cada número- y la falta de especialización de algunos periodistas que les otorgan una credibilidad excesiva a las revistas especializadas.

La proliferación de los suplementos de salud -El Mundo aumentó en 1999 a doce las ocho páginas que publicaba en 1998- y la escasez de periodistas especializados ha generado también que los directores de los periódicos nacionales hayan optado por dedicar a sus periodistas científicos a estos suplementos. Estos, además, han tenido muy buena aceptación publicitaria, pues en ellos se anuncian desde laboratorios farmacéuticos o medicamentos concretos hasta productos de lujo, pues no hay que olvidar que los suplementos especializados son leídos principalmente por los profesionales dedicados a los temas que se publican en ellos. En este sentido, es evidente que los médicos representan un colectivo de alto poder adquisitivo en España.

13 COLOMBO, Furio: Últimas noticias sobre periodismo. Anagrama. Barcelona. 1997. 


\section{Una posible explicación al aumento de suplementos de salud desde 1998}

La aprobación en 1998 de una ley por la cual la Seguridad Social española no financia determinados fármacos que, teniendo las mismas propiedades, valen mucho más caros e, incluso, se recomienda en ella el uso de fármacos genéricos -conocida popularmente como el "medicamentazo"-, ha modificado las estrategias de venta de algunos laboratorios farmacéuticos, los cuales han apostado por los suplementos de salud de los diarios generalistas. Antes publicitaban sus medicamentos entre los médicos, a los cuales les daban diversos regalos si prescribían sus fármacos frente a otros más baratos y a los que convencían entregándoles en sus consultas determinados estudios elaborados por el propio laboratorio y publicados en alguna revista financiada por ellos. Ahora, tras esta ley, han comprobado que la mejor fórmula es publicitar las propiedades de sus productos en los suplementos de diarios generalistas en forma de información.

El lector será quien solicitará a su médico que le recete un determinado medicamento del que ha leído determinadas propiedades. El médico le informará de que no es financiado por la Seguridad Social, pero el paciente, preocupado por su enfermedad y sugestionado por la "noticia" que ha leído, estará dispuesto a pagarlo. Estos laboratorios financian estudios sesgados sobre sus propios fármacos o tratamientos, que luego son publicados en revistas médicas financiadas íntegramente por esas mismas empresas -llama la atención la cantidad de revistas médicas que de forma gratuita reciben los profesionales de la medicina en sus domicilios- y que sirven de tapadera y de enganche para presentar el estudio en los suplementos de salud de los medios de comunicación.

Esta práctica, ya antigua, también se ha potenciado por determinadas normas internas en algunas empresas médicas tanto públicas como privadas, que prohíben o dificultan las entrevistas de los médicos con los visitadores de los laboratorios.

Un ejemplo de esto es el caso del rTPA, un producto que, según los medios de comunicación, aumentaba la supervivencia tras el infarto de miocardio. La realidad, cuando se profundiza en el trabajo, es que un producto que es diez veces más caro que su competidor, eleva la supervivencia tras el ataque cardiaco desde el 92,7\% al 93,7\%. "Nadie duda de que la diferencia es significativa desde el punto de vista matemático, pero no lo es desde el ángulo clínico", señalaba el doctor José Luis de la Serna en el diario El Mundo. Pero es obvio que alguien que acaba de salir de un infarto paga lo que sea por tener el mejor medicamento.

Estas vinculaciones publicitarias tan directas de los suplementos de Salud, que aunque quieran ser camufladas, son, en general, evidentes, está creando un estado de opinión entre algunos periodistas científicos e investigadores sociales en el sentido de que los suplementos de salud pueden llegar a desprestigiar a un periódico, debido a que se pone de manifiesto de forma muy explícita que priman más los intereses comerciales que los informativos. Y existe el peligro de que la 

generalista: el caso de los científicos sanitarios

falta de credibilidad que están teniendo las revistas e investigaciones médicas se extienda al resto de las noticias del periódico.

Algo muy diferente de lo que sucede con los suplementos de ciencia o con la información científica en general, cuyo valor añadido siempre ha sido, por lo menos hasta ahora, el prestigio y la credibilidad. Es decir, en la mente de los dueños de los periódicos que dedican gran espacio a la ciencia nunca ha prevalecido el hecho de divulgar la ciencia, simplemente, para aumentar el conocimiento de la población. Sus decisiones han estado influidas por los estudios de comunicación de masas que demuestran que la información científica traslada los valores de rigor y credibilidad que tiene la ciencia entre la población al resto de las noticias del periódico. Así, cuanto más prestigioso quiera ser un diario, desde The New York Times, hasta Le Monde mayor atención le dedicará, en teoría, a la información científica.

En el caso de la información sobre medicina y salud y, en especial, de los suplementos, habría que estudiar la cantidad de noticias inducidas por los laboratorios farmacéuticos y el tratamiento de determinadas informaciones, así como si existen relaciones entre el laboratorio o el medicamento que aparece como anuncio en una página y la información médica que aparece en ella, para especificar si realmente suponen un desprestigio en cuanto a la credibilidad de un periódico.

Sería, por ejemplo, éticamente impresentable que en una página donde aparezca un artículo sobre la gripe aparezca un anuncio de fármaco antigripal Frenadol. Considero que no se han elaborado suficientes estudios sobre este tema ${ }^{14}$, de reciente implantación en España. Pero como ya he mencionado, puede que el efecto de estos suplementos, a la larga, sea contraproducente para el periódico si no se toman las medidas adecuadas.

No obstante, debe mencionarse que los posibles efectos negativos de la evidente publicidad y de las informaciones inducidas por las empresas que financian la publicidad del suplemento de salud pueden paliarse con el efecto de credibilidad aportado por las noticias de biociencia básica que introducen, sobre todo de genética, y que trasladan la credibilidad científica general al suplemento.

Otro argumento para justificar que los suplementos de salud no desprestigian al periódico que lo publica se centraría, sobre todo, en el hecho, de que

14 Respecto a este asunto considero que debería estudiarse, por ejemplo, la proporción de informaciones médicas por áreas de la medicina y relacionarlas con las áreas de mayor competitividad entre los laboratorios. Así, por ejemplo, se sabe que los fármacos destinados a tratar la hipertensión, las cardiopatías o el cáncer son los más caros del mercado y entre los que existe mayor competitividad empresarial. Los analgésicos, por el contrario, son muy baratos, las marcas están muy consolidadas y no existe demasiada competitividad. Si se demostrara que existe más información publicada sobre fármacos para tratar la hipertensión que sobre los analgésicos, podría representar un punto de partida para reflexionar sobre la manipulación de los suplementos de salud por los laboratorios. Es evidente que este resultado a favor de la hipertensión no se justifica por el interés social, pues el consumo de analgésicos es superior al de fármacos para la hipertensión. 
muchas de las noticias que introducen los suplementos provienen de revistas médicas o especializadas tipo Nature o Science las cuales trasladan también al suplemento su cuota de rigor y credibilidad.

Un estudio riguroso de esas informaciones difundidas por revistas médicas o especializadas posiblemente demostraría que aunque algunas sean realizadas por universidades, detrás existe una financiación de determinadas empresas. Casi todas las investigaciones sobre salud están financiadas por laboratorios farmacéuticos privados, desde la obtención de Dolly, cuya investigación aparecida en Nature fue financiada por al empresa PPL Therapeutics, hasta los más nimios avances sobre el tratamiento del cáncer o el sida.

Esta imbricación de relaciones entre intereses ocultos de los laboratorios y la información que inducen a publicar en los medios no aparece, o al menos no es tan explícita como en el caso de las biociencias, en la información científica relacionada con otras áreas de la ciencia, como podrían ser la astrofísica, la geología o la zoología.

En este sentido, parece obvio que una información sobre el descubrimiento de una nueva galaxia o una nueva explicación de por qué se generan las erupciones volcánicas son noticias "más limpias" en cuanto a posibles intereses oscuros, que todas aquellas relacionadas con la salud.

Otro valor añadido, en este caso, tanto de la publicación de noticias de ciencia como de salud es el interés social de ambas. Los periódicos son cada día más sensibles a este interés así como a las solicitudes de información concreta de los lectores. La iniciativa comenzada por el diario El Mundo en 1999 y El País en 2000 por la cual los lectores que accedan al periódico a través de Internet pueden evaluar cada noticia y señalar cuál ha sido la mejor del día, podrá modificar en un futuro las formas de confección de un periódico. Esto, sin duda, contribuirá a que, posiblemente, se introduzcan más informaciones en la prensa española que ayuden a la divulgación de la ciencia.

\section{Noticias científicas diarias frente a los suplementos}

Desde un punto de vista simplemente de divulgación científica es evidente que lo mejor es que coexistan a la vez las noticias científicas diarias, bien en la sección de sociedad bien en una específica, con los suplementos, que pueden ser semanales o de cualquier otra periodicidad. Ambos sistemas poseen sus ventajas e inconvenientes y, si debe elegirse entre uno de ellos, debe hacerse de forma que el modelo seleccionado se vea reforzado por alguna de las ventajas del que no lo ha sido. A continuación se describen algunas de las características de ambos sistemas.

\section{Opción de incluir noticias científicas diarias}

Es el modelo elegido por Le Monde en Francia y en España $A b c$ y, en menor medida, La Vanguardia. Tiene la ventaja de que se informa a los lectores de los 

generalista: el caso de los científicos sanitarios

acontecimientos el mismo día en que son noticia y no se les retiene información para hacerla coincidir con el día de publicación del suplemento. En principio, tiene el inconveniente de que se dispone de menos tiempo para elaborar la noticia. Sin embargo, en el caso concreto del periodismo científico, este problema no es tan relevante, sobre todo, si consideramos que una parte importante de la información que se publica sobre ciencia proviene de las revistas especializadas que, normalmente, envían el comunicado de prensa a las redacciones con una semana de antelación.

El resto de la información suele ser habitualmente del tipo "no contingente". Es decir, una investigación sobre la contaminación por mercurio del Mediterráneo o sobre la emigración extraña de unas cigüeñas en Castilla y León puede prepararse con tiempo suficiente, pues sus resultados no ocurren en un momento concreto, y publicarse cualquier día de la semana. Normalmente, estas informaciones han sido filtradas al periodista por el científico o ha sido el propio redactor quien ha llamado a sus fuentes habituales y les ha preguntado si saben de algún trabajo interesante que se estuviera realizando en ese momento.

La mayor ventaja de introducir las noticias científicas diariamente junto al resto de la información está en que el lector no se cansa de tanta información científica, como sucede en el caso de los suplementos. La experiencia demuestra que aunque éstos se separen para leerlos con más atención, la vida actual no permite esta dedicación y en la mayoría de los casos la información contenida en el suplemento termina por no leerse. Teniendo en cuenta que los periódicos generalistas deben tener como objetivo un amplio sector de la población que, normalmente, tiene cierto o gran interés pero no es fanática de la información científica, lo mejor para que esta información adquiera difusión es que aparezca en una sección diaria.

Además, la posibilidad de incluir noticias diarias permite informar sobre el protocolo de la ciencia así como de la política científica. Es necesario, al igual que sucede con la política, la economía o la cultura, informar sobre comisiones parlamentarias, desarrollo de congresos o debates en el senado o en el congreso de temas relacionados con la ciencia. Si se relega la información a los suplementos, estos temas no podrían ser publicados, porque, normalmente, pierden interés si no aparecen el día en el que ocurren.

Uno de los problemas que presenta la información científica diaria es la imposibilidad de publicar artículos de revisión sobre un tema concreto. Sin embargo, los suplementos dominicales son una oportunidad magnífica para incluir estos temas. Un problema asociado a la difusión diaria de noticias de ciencia reside en que los responsables de los periódicos ven menos la necesidad de tener redactores especializados y encargan las informaciones a sus redactores de sociedad. Esto es un error, pues esta información diaria debe hacerse con más rapidez, pero sin perder profesionalidad. Un redactor especializado también puede aportar la visión 
científica de un tema de actualidad aunque no sea propiamente información sobre un hallazgo. Desde las causas de un temporal o un terremoto hasta una intoxicación alimentaria como el caso de las dioxinas. Desde unas noticias sobre la cantidad de residuos sólidos que se generan y su influencia en el medio ambiente hasta un suceso producido por un accidente ecológico son temas en los que un redactor especializado en ciencia puede incorporar sus conocimientos para que la sociedad adquiera la vertiente científica de muchos de los problemas cotidianos que acontecen.

Otra posible disfunción de incluir las noticias de ciencia en la sección diaria de sociedad es el aumento de la tendencia a incrementar la espectacularidad, que no sensacionalismo, de las noticias científicas, pues éstas deben competir con las de asesinatos, violaciones o tragedias que, habitualmente, también se insertan en la sección de sociedad.

\section{Opción de los suplementos}

Los mayores defensores de los suplementos específicos sobre información científica son los investigadores. Poco dados a la comunicación y acostumbrados al rigor absoluto en el vocabulario, los suplementos ofrecen una oportunidad de que los artículos, aunque sean escritos por periodistas, contengan una complejidad léxica muy superior, tan del agrado de los científicos españoles, pero de menor nivel de comprensión para el resto de los lectores.

Muchas veces el enganche para publicar una información en un suplemento no es una noticia en sí, sino un nuevo proceso de una investigación o una entrevista de carácter con un científico. Son interesantes, pero la mayoría de los lectores no se sienten atrapados si no hay un titular o unas declaraciones ingeniosas que, habituales en géneros periodísticos como el reportaje, no son utilizadas en los suplementos científicos por el temor a dar la impresión de carecer de rigor. Normalmente, en los suplementos españoles se acepta no utilizar un lenguaje tan periodístico. Esto supone mayor rigor y credibilidad, que, como he mencionado en páginas anteriores, se traslada al resto del periódico, pero influye en el grado de comprensión y, sobre todo, de atracción del texto por parte del lector no especializado.

En cualquier caso, los grandes diarios internacionales, excepto Le Monde, tiene suplementos de ciencia y tecnología. Desde The New York Times, cuyo suplemento Science Times aparece todos los martes desde 1978 y fue el iniciador de esta corriente, hasta The Washington Post o el diario Liberation, aunque el suplemento de éste último trata sobre todo asuntos relacionados con la antropología. Incluso, prestigiosas revistas como The Economist contienen una sección científica fija de varias páginas. 

generalista: el caso de los científicos sanitarios

\section{Fórmula ideal}

A pesar de todo ello, considero que la fórmula ideal sería la publicación de noticias diarias sobre ciencia junto a la publicación de grandes reportajes en los suplementos dominicales, descartando los suplementos especializados.

Sin embargo, también creo que los suplementos científicos en los diarios generalistas deben potenciarse en el momento actual en la ciencia española, pues son los únicos órganos donde los científicos pueden comunicarse en español. A falta de revistas científicas de prestigio y de información general editadas por entidades como la Real Academia de Ciencias o el propio CSIC, que en 1997, con la llegada del nuevo jefe de prensa interrumpió la publicación de la revista Fronteras de la ciencia y la tecnología, los suplementos de ciencia de los diarios generalistas españoles suponen una oportunidad muy buena para que escriban los científicos españoles sobre su visión de la ciencia actual.

Permiten, asimismo, que puedan publicarse resúmenes contados de una manera periodística de lo que ha acontecido en congresos celebrados en sitios cercanos a donde se edita el periódico. Al contrario de lo que sucede con la cultura, en la que un congreso importante es difundido por los medios de comunicación en sus páginas especializadas, la ciencia española no goza de ese privilegio.

El problema fundamental de los suplementos, desde el punto periodístico, estriba en el exceso de información que a veces publican.

A modo de resumen de este trabajo, creo que la fórmula ideal de difusión de la ciencia y sanidad estaría en publicar al menos una o dos noticias diarias, valorando la información internacional pero también contextualizándola al país.

Muchas veces vale más escribir un artículo de recopilación que varios similares aportando sólo un dato. Por ejemplo, ya no es noticia el descubrimiento de un nuevo gen. Sería más oportuno, enganchándonos en el descubrimiento de un nuevo gen, escribir algo sobre qué significa el proyecto del genoma humano y qué implicará en un futuro.

También deberían publicarse entrevistas de carácter y reportajes menos noticiosos pero, más que insertarlos en un suplemento específico, sería conveniente incluirlos en un dominical o en un suplemento de cultura. La ciencia es parte de la cultura e incluirla en ellos ayudaría a prestigiarla.

Asimismo, debe incrementarse la publicación de artículos que ofrezcan una visión social, económica y política de la ciencia.

Respecto al hecho del papel de los suplementos en la difusión de la ciencia española por los propios científicos, creo que ésa no es su función sino que debe hacerse por medio de revistas de prestigio, cuyas conclusiones más interesantes se publiquen a modo de noticia en la información diaria. Revistas españolas que cuenten, incluso, con una gabinete de prensa que elabore comunicados similares a los que realizan Nature y Science. Si esto no se logra, quizá la única manera de 
impulsar la ciencia española en la sociedad sea la vuelta de los suplementos científicos.

Debe mencionarse asimismo que para que las revistas de divulgación de la ciencia española adquieran prestigio, deben ser apoyadas por los poderes públicos. Pero no de forma testimonial ni con buenas intenciones sino con medidas concretas como, por ejemplo, que para obtener una plaza en una universidad española o para conseguir un aumento de sueldo debido a la producción científica tenga tanto valor publicar en una de esas revistas como en las extranjeras.

Cuadro general con los datos sobre dónde están insertadas las noticias de ciencia en los seis diarios españoles estudiados -El País, Abc, El Mundo, El Periódico de Cataluña, La Vanguardia y Diario 16-, de febrero a julio de 1998. La segunda columna indica el número de noticias que aparecen en la sección de sociedad. En la cuarta, se recogen las que se publican en los suplementos de ciencia o de salud.

\begin{tabular}{|l|l|}
\hline $\mathbf{1}$ & Noticias sección sociedad \\
\hline $\mathbf{2}$ & $\%$ Noticias sociedad respecto al total del periódico (ciencia) \\
\hline $\mathbf{3}$ & Noticias suplementos \\
\hline $\mathbf{4}$ & $\%$ Noticias suplemento respecto al total del periódico (ciencia) \\
\hline $\mathbf{5}$ & $\%$ de noticias en cada periódico sobre el total de noticias (ciencia) \\
\hline
\end{tabular}

\begin{tabular}{|c|c|c|c|c|c|c|}
\hline Cabecera & Noticias soc. (1) & \% (2) & Noticias sup. (3) & \% (4) & Total cabecera & \% (5) \\
\hline ABC & 178 & $49,9 \%$ & 179 & $50,1 \%$ & 357 & $24,5 \%$ \\
\hline El País & 73 & $21,4 \%$ & 268 & $78,6 \%$ & 341 & $23,4 \%$ \\
\hline El Mundo & 93 & $23,0 \%$ & 311 & $77,0 \%$ & 404 & $27,7 \%$ \\
\hline La Vanguardia & 153 & $100,0 \%$ & 0 & $0,0 \%$ & 153 & $10,5 \%$ \\
\hline Diario 16 & 110 & $100,0 \%$ & 0 & $0,0 \%$ & 110 & $7,5 \%$ \\
\hline El Periódico & 93 & $100,0 \%$ & 0 & $0,0 \%$ & 93 & $6,4 \%$ \\
\hline \multicolumn{7}{|c|}{116,67} \\
\end{tabular}


186 Los suplementos especializados como guetos de noticias en la prensa generalista: el caso de los científicos sanitarios

\section{Ciencia}

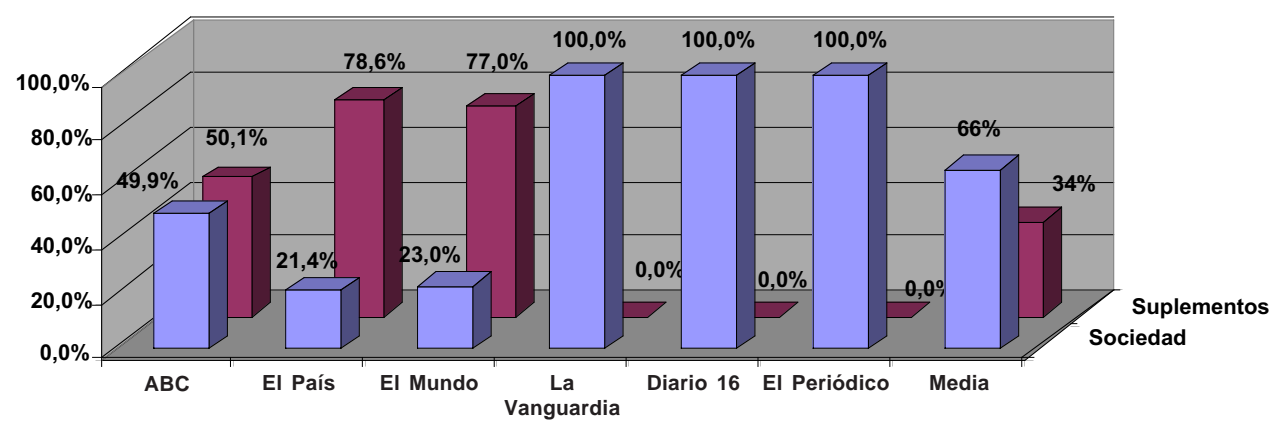

(Recibido el 15-6-2002, aceptado el 5-7-2002) 